\title{
Cerebrospinal Fluid Penetration Rate and Efficacy of Afatinib in Patients with EGFR Mutation-positive Non-small Cell Lung Cancer with Leptomeningeal Carcinomatosis: A Multicenter Prospective Study
}

\author{
AKIHIRO TAMIYA ${ }^{1}$, MOTOHIRO TAMIYA ${ }^{2}$, TAKASHI NISHIHARA ${ }^{2}$, TAKAYUKI SHIROYAMA ${ }^{2}$, \\ KEIKO NAKAO $^{1}$, TAISUKE TSUJI ${ }^{1}$, NAOKO TAKEUCHI ${ }^{1}$, SHUN-ICHI ISA $^{1}$, \\ NAOKI OMACHI $^{1}$, NORIO OKAMOTO ${ }^{2}$, HIDEKAZU SUZUKI ${ }^{2}$, KYOICHI OKISHIO $^{1}$, \\ AYANO IWAZAKI ${ }^{3}$, KIMIE IMAI ${ }^{3}$, TOMONORI HIRASHIMA ${ }^{2}$ and SHINJI ATAGI ${ }^{1}$ \\ ${ }^{1}$ National Hospital Organization Kinki-chuo Chest Medical Center, Sakai, Japan; \\ ${ }^{2}$ Osaka Prefectural Medical Center for Respiratory and Allergic Diseases, Habikino, Japan; \\ ${ }^{3}$ Faculty of Pharmaceutical Sciences, Setsunan University, Hirakata, Japan
}

\begin{abstract}
Background: Afatinib is an effective first-line treatment for epidermal growth factor receptor (EGFR) mutation-positive advanced non-small cell lung cancer (NSCLC). However, few reports have addressed the influence of cerebrospinal fluid (CSF) penetration rate on the efficacy of afatinib in patients with central nervous system metastases. Therefore, we conducted a prospective multicenter trial to evaluate the CSF penetration rate and efficacy of afatinib in patients with EGFR mutation-positive NSCLC with leptomeningeal carcinomatosis. Patients and Methods: Eleven patients with histologically-proven EGFR mutation-positive NSCLC with leptomeningeal carcinomatosis were enrolled in the study between April 2014 and November 2015. They were treated with afatinib (40 mg/day), and blood and CSF levels of afatinib were analyzed on day 8. The primary endpoint was $C S F$ penetration rate. Secondary endpoints included the objective response rate (ORR), progression-free survival (PFS), and overall survival (OS). Results: The median age of patients was 66 years. Five patients harbored an exon 19 deletion, three harbored a p.L858R point mutation, and three harbored an uncommon exon 18 mutation. The levels of afatinib in blood and CSF (mean $\pm S D$ ) were 233.26 $\pm 195.40 \mathrm{nM}$ and 3.16 \pm 1.95
\end{abstract}

Correspondence to: Akihiro Tamiya, MD, Department of Internal Medicine, National Hospital Organization Kinki-chuo Chest Medical Center, 1180 Nagasonecho, Kita-ku, Sakai, Osaka 5918555, Japan. Tel: +81 722523021, Fax: +81 722523041, e-mail: atamiya@kch.hosp.go.jp

Key Words: Afatinib, cerebrospinal fluid penetration rate, epidermal growth factor receptor, leptomeningeal carcinomatosis. $n M$, respectively. The CSF penetration rate was $2.45 \pm 2.91 \%$. The ORR was $27.3 \%$ (three out of 11 patients), and two out of these three responders had uncommon EGFR mutations. The median PFS and OS were 2.0 and 3.8 months, respectively. Conclusion: The median CSF penetration rate of afatinib was higher than previously reported. Afatinib was effective against leptomeningeal carcinomatosis particularly in patients with NSCLC harboring uncommon EGFR mutations. The criteria for selecting a specific EGFR tyrosine kinase inhibitor for therapy of NSCLC should include its ability to penetrate CSF and its efficacy against specific mutation types.

Lung cancer is the leading cause of cancer-related death in many countries (1), and non-small cell lung cancer (NSCLC) accounts for approximately $80 \%$ of all lung cancers .

Central nervous system (CNS) metastases, including brain metastases and leptomeningeal carcinomatosis (LMC), occur commonly in NSCLC (1). Among patients with NSCLC, 10$25 \%$ present with CNS metastases at the time of diagnosis and up to $50 \%$ will develop CNS metastases at some point during the course of their disease $(2,3)$. The development of brain metastases carries a high risk of morbidity and mortality and can negatively impact the quality of life of patients with NSCLC (4).

Epidermal growth factor receptor-tyrosine kinase inhibitors (EGFR-TKIs) (e.g. gefitinib, erlotinib and afatinib) show remarkable inhibitory activity in patients with NSCLC harboring somatic activating mutations of the EGFR gene regardless of the presence of CNS metastases (5-8). However, resistance to EGFR-TKI may eventually develop, and approximately one-third of patients develop CNS metastases after acquiring EGFR-TKI resistance $(9,10)$. 
Afatinib is an orally available, irreversible avian erythroblastosis oncogene $\mathrm{B}$ family blocker that has been approved worldwide, including the US, EU and Japan, for the treatment of patients with EGFR mutation-positive NSCLC (11). The analysis of 81 patients with asymptomatic brain metastases from the LUX-Lung 3 and LUX-Lung 6 studies who were treated with afatinib or cisplatin in combination with pemetrexed/gemcitabine as first-line therapy revealed a median progression-free survival (PFS) of 8.2 and 5.4 months, respectively (12). The results of the compassionate-use program of afatinib indicated that median time to treatment failure for patients with CNS metastasis was 3.6 months and did not differ from a matched group of 100 patients without CNS metastasis (5). Therefore, CNS metastases did not affect the efficacy of afatinib in patients with lung cancer with $E G F R$ mutations. These findings suggest that afatinib could potentially be effective for EGFR mutation-positive NSCLC with CNS metastases in both first- and later-line therapy.

The pharmacokinetics and pharmacological study of the absorption, distribution, metabolism, and excretion of afatinib demonstrated that it was metabolized to a minor extent, and no active metabolites were formed by cytochrome enzymes (13). Therefore, measuring the afatinib concentration in plasma is crucial.

The purpose of this study was to measure the plasma and cerebrospinal fluid (CSF) concentrations of afatinib in patients with NSCLC with LMC harboring active EGFR mutation and to assess the clinical efficacy of afatinib in these patients.

\section{Patients and Methods}

Study design. We conducted a prospective multicenter trial to evaluate the CSF penetration rate and efficacy of afatinib in patients with EGFR mutation-positive NSCLC with LMC. The primary endpoint was CSF penetration rate. Secondary endpoints included the objective response rate (ORR), PFS, and overall survival (OS). All participants provided written informed consent. The study protocol was approved by the appropriate Ethical Committees of each institution. Research was conducted in accordance with the 1964 Declaration of Helsinki and its later amendments. The trial was registered in the University Hospital Medical Information Network Clinical Trials Registry (UMIN000014065).

Patient eligibility. The main inclusion criteria included histologically proven EGFR mutation-positive NSCLC; a confirmation of LMC by CSF or imaging using cystography brain magnetic resonance imaging (MRI), such as fluid attenuated Inversion recovery (FLAIR) subarachnoid space hyperintensity or subcortical low intensity prior to patients' enrollment in the study; age of $\geq 20$ years; no previous afatinib treatment with gefitinib and erlotinib permitted; an Eastern Cooperative Oncology Group performance status (ECOG PS) of 03; adequate bone marrow, liver, and kidney function; and negativity for human immunodeficiency virus, human T-cell lymphotropic virus-1, hepatitis B surface antigen, hepatitis C virus antibody, and Treponema pallidum antibody.
The main exclusion criteria included severe diarrhea, interstitial lung disease on chest X-ray/computed tomography (CT), extensive pleural effusion or ascites, active multiple primary cancer, uncontrolled diabetes mellitus or hypertension, severe heart failure or a history of myocardial infarction/angina in the previous 6 months, serious drug allergy history, and acute inflammatory disease.

Treatment and evaluation. The enrolled patients were treated with afatinib (40 mg/day) until disease progression or the occur $\neg$ rence of unacceptable toxicity.

Within 4 weeks prior to study entry, all patients underwent a bone scan, CT of the chest and abdomen, and MRI of the brain. Within 2 weeks prior to study entry, all patients underwent chest radiography, a complete blood cell count, and blood chemistry measurements, and within 1 week prior to study entry, all patients underwent a physical examination including body weight measurements. In addition, all patients had their ECOG PS assessed and their medical history taken.

Peripheral blood and biochemical examinations were repeated at least once every 2 weeks throughout the course of the study. Chest CT and brain MRI scans were performed to evaluate the efficacy of afatinib after 4-8 weeks of treatment. Following the completion of the treatment cycle, chest CT and brain MRI scans were performed at 8 week intervals. The overall efficacy of afatinib was evaluated using Response Evaluation Criteria in Solid Tumors, version 1.1 (14).

PFS was defined as the time interval between the date of enrollment and the date of confirmation of disease progression or the date of death from any cause. OS was defined as the time interval between the date of enrollment and the date of death from any cause. Patients with missing data were censored at the date of their last PFS assessment. The CSF penetration rate was defined as the concentration of afatinib in CSFrelative to its concentration in plasma.

Test methods. Blood and CSF samples were collected from the enrolled patients. The trough blood and CSF concentrations of afatinib were measured on day 8 after administration. Blood collected in potassium ethylenediaminetetra-acetic acid-containing tubes was immediately centrifuged at $1,800 \times g\left(4^{\circ} \mathrm{C}\right)$ for 10 minutes. Plasma and CSF samples were stored at $-80^{\circ} \mathrm{C}$ until assayed.

The plasma (1.5-2.6 ml) and CSF (2.3-4.9 ml) samples were processed by solid-phase extraction according to the method of Stopfer et al. (13). with modifications. Briefly, $10 \mathrm{ml}$ of acetonitrilewater ( $90: 10$ by volume) was used as rinsing medium after the sample was applied to the cartridge column (Discovery ${ }^{\circledR}$ DSC18LT; $2 \mathrm{~g}, 12 \mathrm{ml}$; SUPELCO, Bellefonte, PA, USA) and the absorbed material was eluted as described previously (13). The eluate was evaporated to dryness under a stream of nitrogen at $45^{\circ} \mathrm{C}$ and the residue was dissolved in methanol (200 $\mu$ l for plasma and $100 \mu \mathrm{l}$ for CSF). An aliquot of the solution was applied to a high performance liquid chromatography column to measure the concentration of afatinib. The recovery was $95.3-97.9 \%$. The highperformance liquid chromatography system (JASCO Corp., Tokyo, Japan) comprised a PU-980 pump, UV-970 (plasma) or UV-1570 ultraviolet detector equipped with a semi-micro cell unit (CSF), 807-IT integrator, and U-620 column heater (Sugai Chemical Ind. Co., Ltd., Wakayama, Japan).

Separation was performed using Inertsil ODS-2 (particle size, 5 $\mu \mathrm{m})$ columns, $3.0 \mathrm{~mm}$ I.D. $\times 150 \mathrm{~mm}$ for plasma, and $2.1 \mathrm{~mm}$ I.D. $\times 150 \mathrm{~mm}$ for CSF (GL Sciences Inc., Tokyo, Japan). The mobile phase was a 55:44.5:0.5 mixture of $0.1 \mathrm{M}$ ammonium acetate 
Table I. Patient characteristics. All patients had adenocarcinoma.

\begin{tabular}{|c|c|c|c|c|c|c|c|}
\hline \multirow[b]{2}{*}{ Patient no. } & \multicolumn{7}{|c|}{$E G F R$ mutation status } \\
\hline & Age (years) & Gender & ECOG PS & At diagnosis & From CSF & $\begin{array}{l}\text { No. of previous } \\
\text { CTX lines }\end{array}$ & $\begin{array}{c}\text { Previous } \\
\text { EGFR-TKI use }\end{array}$ \\
\hline 1 & 65 & $\mathrm{~F}$ & 3 & Ex21 L858R & N.E & 2 & Yes \\
\hline 2 & 74 & M & 3 & Ex19 DEL & N.E & 3 & Yes \\
\hline 3 & 51 & M & 1 & Ex19 DEL & Ex19 DEL & 1 & Yes \\
\hline 4 & 63 & $\mathrm{~F}$ & 3 & Ex19 DEL & - & 0 & No \\
\hline 5 & 57 & F & 1 & Ex21 L858R & Ex21 L858R & 4 & Yes \\
\hline 6 & 50 & $\mathrm{~F}$ & 2 & Ex18 G719A & N.E & 3 & Yes \\
\hline 7 & 74 & $\mathrm{~F}$ & 1 & Ex21 L858R & Ex21 L858R & 2 & Yes \\
\hline 8 & 68 & M & 1 & Ex18 G719S & N.E & 4 & Yes \\
\hline 9 & 79 & $\mathrm{~F}$ & 3 & Ex19 DEL & N.E & 2 & Yes \\
\hline 10 & 68 & $\mathrm{~F}$ & 2 & Ex18 G719S & Ex18 G719S & 3 & Yes \\
\hline 11 & 66 & $\mathrm{~F}$ & 2 & Ex19 DEL & N.E & 2 & No \\
\hline
\end{tabular}

CSF, Cerebrospinal fluid; CTX, chemotherapy; DEL, deletion; ECOG, Eastern Cooperative Oncology Group; EGFR, epidermal growth factor receptor; Ex, exon; F, female; M, male; N.E, not evaluated; PS, performance status; TKI, tyrosine kinase inhibitor.

(adjusted at $\mathrm{pH} 8.5$ ), acetonitrile, and triethylamine. The flow rate was 0.4 and $0.2 \mathrm{ml}$ per minute for plasma and CSF, respectively. The column temperature was set at $30^{\circ} \mathrm{C}$. Afatinib from SYN kinase (Melbourne, Australia) was used as a standard and was detected at a retention time of approximately 13 minutes by absorbance at $254 \mathrm{~nm}$. The lower limit of quantification was $0.5 \mathrm{ng}$ (equivalent to $2.0 \mathrm{ng} / \mathrm{ml}$ ) for plasma and $120 \mathrm{pg}$ (equivalent to $0.35 \mathrm{ng} / \mathrm{ml}$ ) for CSF; the calibration curve was linear up to $100 \mathrm{ng}$ and $5 \mathrm{ng}$, respectively.

Statistical analysis. Continuous variables were analyzed using Student's $t$-test. The results are expressed as means \pm standard deviations (SD). A correlation analysis between the CSF concentration of afatinib and its curative effect was performed using analysis of variance. Survival curves were constructed using the Kaplan-Meier method. The incidence of adverse events was calculated and the distribution of the best overall response was summarized in patients with target lesions. All statistical analyses were conducted using Statistical Package for the Social Sciences for Windows, software version 13.0 (SPSS Inc., Chicago, IL, USA).

\section{Results}

Patients characteristics. In total, 11 patients were enrolled from two institutions between April 2014 and November 2015. The median follow-up time was 3.8 months. A summary of the patients' characteristics is presented in Table I. The median age was 66 (range $=55-79$ ) years. The histological classification of tumors for all patients was adenocarcinoma. Regarding EGFR mutation status, five patients harbored an exon 19 deletion, three harbored a p.L858R point mutation, and three harbored an uncommon exon 18 mutation. Four patients had an ECOG PS of 1, three patients had an ECOG PS of 2, and fur had an ECOG PS of 3. Since all patients in this study had LMC, their physical status was poorer than those enrolled in other clinical trials. One patient received afatinib as first-line therapy, one patient received afatinib as second-line therapy, four received afatinib as third-line therapy, and five received afatinib as fourth or later line of therapy. In addition, nine out of 11 patients were administered other EGFR-TKIs prior to afatinib therapy.

Furthermore, we examined the EGFR mutation status from CSF before afatinib treatment in four patients and none of these patients harbored the p.T790M TKI-resistance point mutation in exon 20 .

Blood and CSF levels of afatinib and CSF penetration rate. The levels of afatinib in blood and CSF were analyzed in 10 and 8 patients, respectively. Table II presents of afatinib in each patient's blood and CSF, as well as the CSF penetration rate of afatinib. The blood and CSF levels of afatinib $($ mean \pm SD) were $233.26 \pm 195.40 \mathrm{nM}$ and $3.16 \pm 1.95 \mathrm{nM}$, respectively. The CSF penetration rate $($ mean \pm SD) was $2.45 \pm 2.91 \%$.

Efficacy. The efficacy of afatinib during the total treatment period is presented in Table II. Three out of 11 patients were still alive at the time of analysis, with a median follow-up time of 3.8 months. The median PFS and OS for the wide group of patients were 2.0 [95\% confidence interval $(95 \%$ $\mathrm{CI})=0.6-5.8]$ months and $3.8(95 \% \mathrm{CI}=1.1-13.1)$ months, respectively. The ORR was $27.3 \%$. Two out of three patients harboring an uncommon EGFR mutation achieved a partial response, while only one out of eight patients harboring an active EGFR mutation showed similar response. This single 
Table II. Concentration of afatinib in plasma and cerebrospinal fluid (CSF), penetration rate, and efficacy in patients with epidermal growth factor receptor (EGFR) mutation-positive non-small cell lung cancer with leptomeningeal carcinomatosis.

\begin{tabular}{|c|c|c|c|c|c|c|}
\hline \multirow[b]{2}{*}{ Patient no. } & \multicolumn{2}{|c|}{ Concentration (nM) } & \multirow[b]{2}{*}{ Penetration rate $(\%)$} & \multirow[b]{2}{*}{ Best response } & \multirow[b]{2}{*}{ PFS (days) } & \multirow[b]{2}{*}{ OS (days) } \\
\hline & Plasma & CSF & & & & \\
\hline 1 & 202.1 & 1.9 & 0.9 & $\mathrm{PD}$ & 45 & 57 \\
\hline 2 & 141.1 & 2.5 & 1.7 & $\mathrm{PD}$ & 32 & 32 \\
\hline 3 & 156.6 & 3.3 & 2.1 & $\mathrm{PD}$ & 44 & 115 \\
\hline 4 & 235.8 & 3.9 & 1.6 & $\mathrm{PD}$ & 19 & $512^{\mathrm{b}}$ \\
\hline 5 & 257.2 & $\mathrm{NE}$ & NE & PD & 67 & 67 \\
\hline 6 & 146.9 & NE & $\mathrm{NE}$ & PR & 309 & 396 \\
\hline 7 & 62.6 & 5.8 & 9.3 & $\mathrm{SD}$ & 176 & 410 \\
\hline 8 & 192.0 & 6.0 & 3.1 & $\mathrm{PD}$ & 61 & 212 \\
\hline 9 & $\mathrm{NE}$ & $\mathrm{NE}$ & NE & PD & 17 & 31 \\
\hline 10 & 767.6 & 0.8 & 0.1 & PR & $171^{\mathrm{a}}$ & $171^{\mathrm{b}}$ \\
\hline 11 & 170.8 & 1.2 & 0.8 & PR & 105 & 105 \\
\hline Mean \pm SD & $233.3 \pm 195.4$ & $3.2 \pm 1.9$ & & & & \\
\hline Median $(95 \% \mathrm{CI})$ & 181.4 & 2.9 & $1.7(2.5 \pm 2.9)$ & - & $61(18-174)$ & $115(32-410)$ \\
\hline
\end{tabular}

NE, Not evaluated; OS, overall survival; PD, progressive disease; PFS, progression-free survival; PR, partial response; SD, stable disease/standard

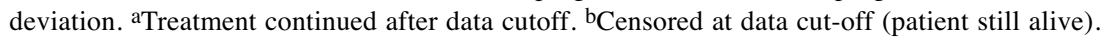

patient did not receive EGFR-TKI treatment before afatinib therapy. In fact, none of the patients harboring active EGFR mutation who had received prior EGFR-TKI treatment achieved a tumor response.

Additional treatment after afatinib therapy. Seven out of 11 patients were unable to receive additional treatment after afatinib therapy due to disease progression and a poor ECOG PS. In one patient, EGFR-TKI was switched the from afatinib to erlotinib and in three patients, afatinib therapy was continued after disease progression.

\section{Discussion}

EGFR-TKIs have essential hydrogen-bond donors and acceptors. These characteristics can affect the penetration of the blood-brain barrier, and representative EGFR-TKIs (e.g. gefitinib, erlotinib, afatinib, and osimertinib) are known to be substrates for P-glycoprotein 1. Therefore, these EGFR-TKIs hardly penetrate the blood-brain barrier (15). Although the CSF levels of some EGFR-TKIs in patients with CNS metastases have been reported, limited data with afatinib are available.

In this study, in order to evaluate CSF penetration of afatinib, we examined the blood and CSF concentrations of afatinib in patients with NSCLC who were diagnosed with LMC from CSF cytology or cystography MRI of the brain, at one time point, immediately before afatinib administration on day 8 . This particular time point was chosen for the following reasons: i) afatinib plasma concentration reaches a steady state by day 8 at the latest (16), and ii) a preclinical pharmacokinetic study using a PC-9 brain metastasis mouse model demonstrated that the concentrations of afatinib in the CSF and plasma were wellcorrelated $(\mathrm{r}=0.844, p<0.01)(17)$. In this study, the blood and CSF levels of afatinib (mean \pm SD) were $233.3 \pm 195.4 \mathrm{nM}$ and $3.2 \pm 1.9 \mathrm{nM}$, respectively. The CSF penetration rate (mean $\pm \mathrm{SD})$ was $2.5 \pm 2.9 \%$. The CSF penetration rate and CSF concentrations of other EGFR-TKIs have been assessed. Togashi et al. reported that the CSF penetration rate of erlotinib $($ mean \pm SD) and gefitinib (mean \pm SD) were $2.77 \pm 0.45 \%$ and $1.13 \pm 0.36 \%$, respectively (18). In the AURA-1 extension study and the AURA-2 study, CSF concentrations of osimertinib in two patients, one patient from each study, were shown to be 0.77 and $3.44 \mathrm{nM}(19,20)$. It is important to note that in each of these studies, different methods of analysis were used for determining CSF concentrations, while the same analysis method was used for determining the CSF penetration rate. Therefore, only the CSF penetration rate of different EGFRTKIs can be directly compared.

Regarding the efficacy of afatnib in this study, the median PFS and median OS were 2.0 (95\% CI=0.6-5.8) months and $3.8(95 \% \mathrm{CI}=1.1-13.1)$ months, respectively. The ORR was $27.3 \%$. Even in later-line use, two out of three patients (no. 6, 8, and 10) harboring an uncommon exon $18 E G F R$ mutation achieved a partial response despite receiving more than three prior TKI treatments. On the other hand, patient 4, with deletion 19 of EGFR, a common mutation, who received afatinib as first- line therapy did not show any response despite sufficient afatinib concentration in CSF.

Investigating the sensitivity of certain mutation types to EGFR-TKIs and the CSF penetration rate of these drugs, and 
dissecting the mechanisms of intrinsic and acquired EGFRTKI resistance would allow us to better predict the clinical efficacy of EGFR-TKIs in patients with LMC. Direct comparison of sensitivity of common and uncommon mutations to EGFR-TKIs to was conducted by Kobayashi and Mitsudomi. The results revealed that there was only a small difference in the half maximal $(50 \%)$ inhibitory concentration $\left(\mathrm{IC}_{50}\right)$ among TKIs for common mutation as shown using $\mathrm{Ba} / \mathrm{F} 3$ deletion 19 -mutant cells (4.8 $\mathrm{nM}$ for elrotinib, $4.8 \mathrm{nM}$ for gefitinib, $1.1 \mathrm{nM}$ for osimertinib and $0.9 \mathrm{nM}$ for afatinib). However, in cells with uncommon mutation (Ba/F3 G719Amutant cells), large differences in $\mathrm{IC}_{50}$ among TKIs were observed (167 nM for elrotinib, $213 \mathrm{nM}$ for gefitinib, $53 \mathrm{nM}$ for osimertinib and $0.9 \mathrm{nM}$ for afatinib) (21).

Elucidating the mechanisms of acquired resistance to previous TKI, especially in later lines of therapy is critically important. The major mechanism of acquired resistance is through the gatekeeper p.T790M point mutation of EGFR, and this mutation is observed in approximately $50 \%$ of patients with NSCLC harboring EGFR mutation who failed to respond to EGFR-TKI (22). It is well known that patients with EGFRp.T790M point mutation are sensitive to osimertinib (21). However, our results are consistent with those of Hata et al. who reported that EGFR-p.T790M point mutation occurs less frequently in those with leptomeningeal metastatic lesions than in those with extracranial lesions (23). Several mechanisms for acquired resistance have been identified in extracranial lesions [e.g., mesenchymal-epithelial transition factor (MET) amplification, human epidermal growth factor receptor type2 (HER2) amplification, and V-raf murine sarcoma viral oncogene homolog B1 (BRAF) mutation], and these mechanisms may contribute to drug resistance in leptomeningeal lesions. Therefore, to overcome acquired drug resistance in patients with EGFR mutation-positive NSCLC with LMC, alternative treatment strategies such as combination therapy of EGFR-TKIs with drugs targeting other molecules could be useful.

\section{Conclusion}

The median CSF penetration rate of afatinib in this study was higher than that reported previously. The efficacy of afatinib for LMC from NSCLC was demonstrated particularly in patients harboring uncommon EGFR mutations. The ability of an EGFR-TKI to penetrate the CSF and its efficacy against tumors with particular mutation types should be considered when deciding which EGFR-TKI to use for therapy.

\section{Acknowledgements}

The Authors wish to thank Mr. S. Fujii and Ms. M. Yamamoto of Setsunan University (Osaka, Japan) for their excellent technical assistance. The Authors also wish to thank all the participants of this study. Additionally, the Authors wish to thank Editage (www.editage.jp) for English language editing.

\section{Funding}

This research was not supported by any specific grant from funding agencies in the public, commercial, or non-profit sectors.

\section{Conflicts of Interest}

A. Tamiya received a grant from Ono Pharmaceutical, BristolMyers Squibb, and personal fees from Boehringer Ingelheim, Eli Lilly, Chugai Pharmaceutical, AstraZeneca, Ono Pharmaceutical, and Bristol-Myers Squibb. M. Tamiya received a grant from Ono Pharmaceutical, Bristol-Myers Squibb, and personal fees from Boehringer Ingelheim, Chugai Pharmaceutical, Pfizer, AstraZeneca, Taiho Pharmaceutical, Eli Lilly, Asahi Kasei Pharmaceutical, Daichi Sankyo Co. Ltd., and Alere Medical. T. Shiroyama received personal fees from Boehringer Ingelheim, Taiho Pharmaceutical, Ono Pharmaceutical. H. Suzuki received personal fees from Boehringer Ingelheim, Eli Lilly, Chugai Pharmaceutical, TAIHO Pharmaceutical. K. Okishio received personal fees from Boehringer Ingelheim, Ono Pharmaceutical. T. Hirashima received a grant from Eli Lilly, Daiichi-Sankyo, Kyowa-Hakko-Kirin, MSD, Merck Serono Co., Ltd, Takeda, TAIHO Pharmaceutical, and personal fees from Boehringer Ingelheim, Bayer Pharmaceutical, and Pfizer. S. Atagi received a grant from Boehringer Ingelheim, Chugai Pharmaceutical, Pfizer, Merck Serono Co., Ltd, Astra Zeneca, Taiho Pharmaceutical, Yakult Pharmaceutical Industry, Eli Lilly and Ono Pharmaceutical, and personal fees from Boehringer Ingelheim, Chugai Pharmaceutical, Astra Zeneca, Taiho Pharmaceutical, Hisamitsu Pharmaceutical Co, Bristol-Myers Squibb and Eli Lilly.

\section{References}

1 Nayak L, Lee EQ and Wen PY: Epidemiology of brain metastases. Curr Oncol Rep 14: 48-54, 2012.

2 Dawe DE, Greenspoon JN and Ellis PM: Brain metastases in non-small-cell lung cancer. Clin Lung Cancer 15: 249-257, 2014.

3 Metro G, Chiari R, Ricciuti B, Rebonato A, Lupattelli M, Gori S, Bennati C, Castrioto C, Floridi P, Minotti V, Chiarini P and Crino L: Pharmacotherapeutic options for treating brain metastases in non-small cell lung cancer. Expert Opin Pharmacother 16: 2601-2613, 2015.

4 Peters S, Bexelius C, Munk V and Leighl N: The impact of brain metastasis on quality of life, resource utilization and survival in patients with non-small-cell lung cancer. Cancer Treat Rev 45: 139-162, 2016.

5 Hoffknecht P, Tufman A, Wehler T, Pelzer T, Wiewrodt R, Schutz M, Serke M, Stohlmacher-Williams J, Marten A, Maria Huber R, Dickgreber NJ and Afatinib Compassionate Use C: Efficacy of the irreversible ERBB family blocker afatinib in epidermal growth factor receptor (EGFR) tyrosine kinase inhibitor (TKI)-pretreated non-small-cell lung cancer patients with brain metastases or leptomeningeal disease. J Thorac Oncol 10: 156-163, 2015.

6 Zhang J, Yu J, Sun X and Meng X: Epidermal growth factor receptor tyrosine kinase inhibitors in the treatment of central nerve system metastases from non-small cell lung cancer. Cancer Lett 351: 6-12, 2014. 
7 Namba Y, Kijima T, Yokota S, Niinaka M, Kawamura S, Iwasaki T, Takeda Y, Kimura H, Okada T, Yamaguchi T, Nakagawa M, Okumura $\mathrm{Y}$, Maeda $\mathrm{H}$ and Ito $\mathrm{M}$ : Gefitinib in patients with brain metastases from non-small-cell lung cancer: review of 15 clinical cases. Clin Lung Cancer 6: 123-128, 2004.

8 Heon S, Yeap BY, Britt GJ, Costa DB, Rabin MS, Jackman DM and Johnson BE: Development of central nervous system metastases in patients with advanced non-small cell lung cancer and somatic EGFR mutations treated with gefitinib or erlotinib. Clinical cancer research 16: 5873-5882, 2010.

9 Eichler AF, Kahle KT, Wang DL, Joshi VA, Willers H, Engelman JA, Lynch TJ and Sequist LV: EGFR mutation status and survival after diagnosis of brain metastasis in nonsmall cell lung cancer. Neuro Oncol 12: 1193-1199, 2010.

10 Omuro AM, Kris MG, Miller VA, Franceschi E, Shah N, Milton DT and Abrey LE: High incidence of disease recurrence in the brain and leptomeninges in patients with nonsmall cell lung carcinoma after response to gefitinib. Cancer 103: 2344-2348, 2005.

11 Li D, Ambrogio L, Shimamura T, Kubo S, Takahashi M, Chirieac LR, Padera RF, Shapiro GI, Baum A, Himmelsbach F, Rettig WJ, Meyerson M, Solca F, Greulich $\mathrm{H}$ and Wong KK: BIBW2992, an irreversible EGFR/HER2 inhibitor highly effective in preclinical lung cancer models. Oncogene 27: 47024711, 2008.

12 Schuler M, Wu YL, Hirsh V, O’Byrne K, Yamamoto N, Mok T, Popat S, Sequist LV, Massey D, Zazulina V and Yang JC: Firstline afatinib versus chemotherapy in patients with non-small cell lung cancer and common epidermal growth factor receptor gene mutations and brain metastases. J Thorac Oncol 11: 380-390, 2016.

13 Stopfer P, Marzin K, Narjes H, Gansser D, Shahidi M, Uttereuther-Fischer $\mathrm{M}$ and Ebner T: Afatinib pharmacokinetics and metabolism after oral administration to healthy male volunteers. Cancer Chemother Pharmacol 69: 1051-1061, 2012.

14 Eisenhauer EA, Therasse P, Bogaerts J, Schwartz LH, Sargent D, Ford R, Dancey J, Arbuck S, Gwyther S, Mooney M, Rubinstein L, Shankar L, Dodd L, Kaplan R, Lacombe D and Verweij J: New response evaluation criteria in solid tumours: revised RECIST guideline (version 1.1). Eur J Cancer 45: 228247,2009 .
15 Heffron TP: Small molecule kinase inhibitors for the treatment of brain cancer. J Med Chem 59: 10030-10066, 2016.

16 Wind S, Schmid M, Erhardt J, Goeldner RG and Stopfer P: Pharmacokinetics of afatinib, a selective irreversible ERBB family blocker, in patients with advanced solid tumours. Clinical Pharmacokinetics 52: 1101-1109, 2013.

17 Zhang SR, Zhu LC, Jiang YP, Zhang J, Xu RJ, Xu YS, Xia B and Ma SL: Efficacy of afatinib, an irreversible ErbB family blocker, in the treatment of intracerebral metastases of non-small cell lung cancer in mice. Acta Pharmacol Sin 38: 233-240, 2017.

18 Togashi Y, Masago K, Masuda S, Mizuno T, Fukudo M, Ikemi Y, Sakamori Y, Nagai H, Kim YH, Katsura T and Mishima M: Cerebrospinal fluid concentration of gefitinib and erlotinib in patients with non-small cell lung cancer. Cancer Chemother Pharmacol 70: 399-405, 2012.

19 Pareek V, Welch M, Ravera E, Zampolin RL, Sequist LV and Halmos B: Marked differences in CNS activity among EGFR inhibitors: case report and mini-review. J Thorac Oncol 11: e135-e139, 2016.

20 Ahn MJ, Tsai CM, Yang JCH. AZD 9291 activity in patients with EGFR-mutant advanced non- small cell lung cancer and brain metastasis: data from phase II studies. Eur J Cancer 51: 3083, 2015.

21 Kobayashi Y and Mitsudomi T: Not all epidermal growth factor receptor mutations in lung cancer are created equal: Perspectives for individualized treatment strategy. Cancer Sci 107: 11791186, 2016.

22 Kobayashi S, Boggon TJ, Dayaram T, Janne PA, Kocher O, Meyerson M, Johnson BE, Eck MJ, Tenen DG and Halmos B: EGFR mutation and resistance of non-small-cell lung cancer to gefitinib. N Engl J Med 352: 786-792, 2005.

23 Hata A, Masago K, Katakami N, Imai Y and Yatabe Y: Spatiotemporal T790M heterogeneity in a patient with EGFRmutant non-small-cell lung cancer. J Thorac Oncol 9: e64-65, 2014. 